\title{
Mode shape and dispersion relation of bending waves in thin silicon membranes
}

\author{
Reimar Waitz, ${ }^{*}$ Stephan Nößner, Michael Hertkorn, Olivier Schecker, ${ }^{\dagger}$ and Elke Scheer \\ Universität Konstanz, D-78464 Konstanz, Germany
}

\begin{abstract}
We study the vibrational behavior of silicon membranes with a thickness of a few hundred nanometers and macroscopic lateral size. A piezo is used to couple in transverse vibrations, which we monitor with a phase-shift interferometer using stroboscopic light. The observed wave pattern of the membrane deflection is a superposition of the mode corresponding to the excitation frequency and several higher harmonics. Using a Fourier transformation in time, we separate these contributions and image up to the eighth harmonic of the excitation frequency. With this method we determine the dispersion relation of membrane oscillations in a frequency range up to $12 \mathrm{MHz}$. We develop a simple analytical model combining stress of a membrane and bending of a thin plate that describes both the experimental data and finite-elements simulations very well. We derive correction terms to account for a finite curvature of the membrane and for the inertia of the surrounding atmosphere. A simple criterion for the transition between stressed membrane and thin plate behavior is presented.
\end{abstract}

PACS number(s): 46.40.Cd, 46.70.De, 46.80. $+\mathrm{j}, 62.30 .+\mathrm{d}$

\section{INTRODUCTION}

Nowadays micromachined membranes are standard parts for a variety of technological applications. They are used as micro hotplates for gas sensors, ${ }^{1}$ as vacuum windows for ion beams, $x$ rays, and ultraviolet radiation, ${ }^{2}$ and as an electronpermeable substrate for transmission-electron microscopy. ${ }^{3}$ In fundamental research they are used as building blocks for photonic crystals, ${ }^{4,5}$ as an elastic substrate for mechanically controlled metallic contacts, ${ }^{6}$ as temperature sensors with high thermal, spacial and temporal resolution, ${ }^{7}$ as ion detectors for mass spectrometry, ${ }^{8}$ and as a sieve on a molecular length scale. ${ }^{9}$ Membranes inside an optical cavity allow coherent coupling of optical and mechanical degrees of freedom. This opens fascinating possibilities for studying the boundary between classical and quantum physics. ${ }^{10,11}$ As an example, laser cooling of vibrational modes from room temperature down to $7 \mathrm{mK}$ has been reported recently. ${ }^{10}$

This broad range of applications motivated studies of the mechanical properties. When the dimensions of a system are reduced to sizes comparable with the phonon wavelength, the discrete nature of the acoustic spectrum becomes visible. For silicon membranes with a thickness of a few hundred nanometers and lateral sizes of about $1 \mathrm{~mm}$, thickness oscillations at tens to hundreds of gigahertz have been studied using time-resolved optical pump probe measurements ${ }^{12}$ and Raman scattering. ${ }^{13}$ At lower frequencies of about $1 \mathrm{MHz}$, the discretization of bending waves in the lateral direction is the dominating mechanism. Although several of the applications mentioned above make use of these excitations, no experimental analysis of mode shape and dispersion relation has been reported so far. As we will show, the bending-wave regime is best described by drum-head (stressed membrane) oscillations for low frequencies, while higher frequencies correspond to thin-plate bending waves. The transition frequency between those regimes depends on the thickness and the prestress of the membrane, and lies in the range of $5 \mathrm{MHz}$ for the membranes used here.

Although the length scale of our membranes is much larger, many aspects discussed here might be interesting for the graphene community. Graphene has a finite bending stiff- ness, shows an inhomogeneous curvature due to spontaneous rippling ${ }^{14}$ and in many experiments it is prestressed because of the pinning to a substrate. A dispersion relation for bending waves taking all these effects into account is presented in this paper.

\section{EXPERIMENT}

We developed an approach to determine the dispersion relation of bending waves in membranes, plates and shells, starting with the measured real-space motion.

Using an imaging interferometer, we detect the surface profile of reflecting samples with subnanometer resolution in the vertical direction and submicrometer resolution in the lateral direction. ${ }^{15}$ Using stroboscopic light, we take subsequent snapshots of the vibrating sample at fixed phases of the oscillation. Using this method we directly measure the deflection of a membrane as a function of space and time.

\section{A. Sample fabrication}

The membranes are fabricated from a silicon-on-insulator (SOI) wafer, using a wet etching process adapted from Ref. 16. The wafer consists of a 340-nm thin silicon layer on top of $400 \mathrm{~nm}$ of silicon oxide, $500 \mu \mathrm{m}$ of bulk silicon, and a silicon nitride etch mask on the backside. Using anisotropic etching in $\mathrm{KOH}$, a hole is etched through an opening in the nitride mask while the top side of the wafer is kept dry by a special etch cell. After 24 hours the $\mathrm{KOH}$ solution reaches the silicon oxide etch-stop layer. Using hydrofluoric acid the oxide is removed, providing a 340-nm-thick silicon membrane with lateral sizes between 200 and $700 \mu \mathrm{m}$ in a rectangular frame made of SOI material. The flatness of the membrane is bettèr than $1 \%$ of the lateral size and is best immediately after oxide removal. The static buckling can be explained by oxide formation on the surface leading to a compressive stress. ${ }^{17,18}$

\section{B. Mechanical control}

The chip is glued to a piezo ring and the backside is connected to a pressure controller and a pump (Fig. 1). We 


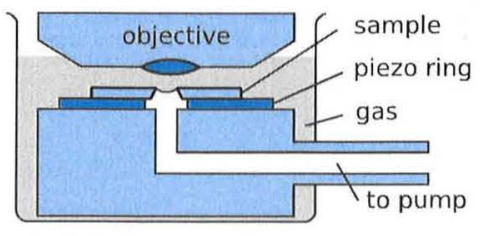

FIG. 1. (Color online) Sketch of the experiment showing schematically the mounting of the sample in the imaging interferometer. The interferometer is indicated by the objective lens. The sample is glued to a piezo ring used to excite the vibrations. With the help of a pump, a pressure difference is applied between upper and lower sides of the sample. It is possible to fill the chamber containing the sample with a heavy gas atmosphere as indicated by the green (light gray) shaded area.

control the static stress by applying a pressure difference between top and bottom sides of the membrane.

Applying an $\mathrm{AC}$ voltage, thickness oscillations of the piezo ring are used to excite vibrations of the membrane. With this simple excitation mechanism we easily obtain large vibration amplitudes up to hundreds of nanometers without damaging the membranes. However, the details of the excitation, given by the coupled resonances of piezo, silicon chip, and other parts, are difficult to unravel. This is problematic when studying any system response amplitudes as a function of the excitation frequency, since it is not straightforward to distinguish resonances of the membrane and resonances of the excitation system. Simulations addressing this issue have been performed and will be published elsewhere. Fortunately this knowledge is not necessary for the experimental approach presented in this paper, as we will explain below.

Above the sample, a microscope objective with an integrated Mirau interferometer detects the reflected light. The range of excitation frequencies from $100 \mathrm{~Hz}$ to $2 \mathrm{MHz}$ is limited by the refresh rate of the CCD camera and the switching time of the stroboscopic light source.

\section{Data analysis}

In general, the observed wave pattern of the membrane deflection will be a superposition of the static profile, the mode corresponding to the excitation frequency $\omega_{e x}$, and several higher harmonics of $\omega_{e x}$. The main reason for the excitation of harmonics can be found in the equation of motion. As we will explain in Sec. III, it is only linear for very small amplitudes. The amplitudes in our experiments $(\sim 100 \mathrm{~nm})$ are small but in this context not negligible compared to the static deflection (a few micrometers). Using a Fourier transformation in time, it is possible to separate different frequency contributions. This way frequency eigenmodes $\tilde{z}\left(x, y, \omega=n \omega_{e x}\right)$ up to the $n=8$ harmonic of the excitation can be imaged, limited by the time resolution of the stroboscopic light source. Examples of such eigenmodes are shown in Figs. 2(a)-2(d) (see figure caption for color code).

In a second step, $\tilde{z}$ is decomposed into a Fourier series [Eq. (1)] to obtain the $\vec{k}$-space representation $c_{l m}$ (Fig. 3) of the mode:

$$
\tilde{z}(x, y, \omega)=\sum_{l, m=1}^{\infty} c_{l m}(\omega) \sin \left(k_{x l} x\right) \sin \left(k_{y m} y\right)
$$
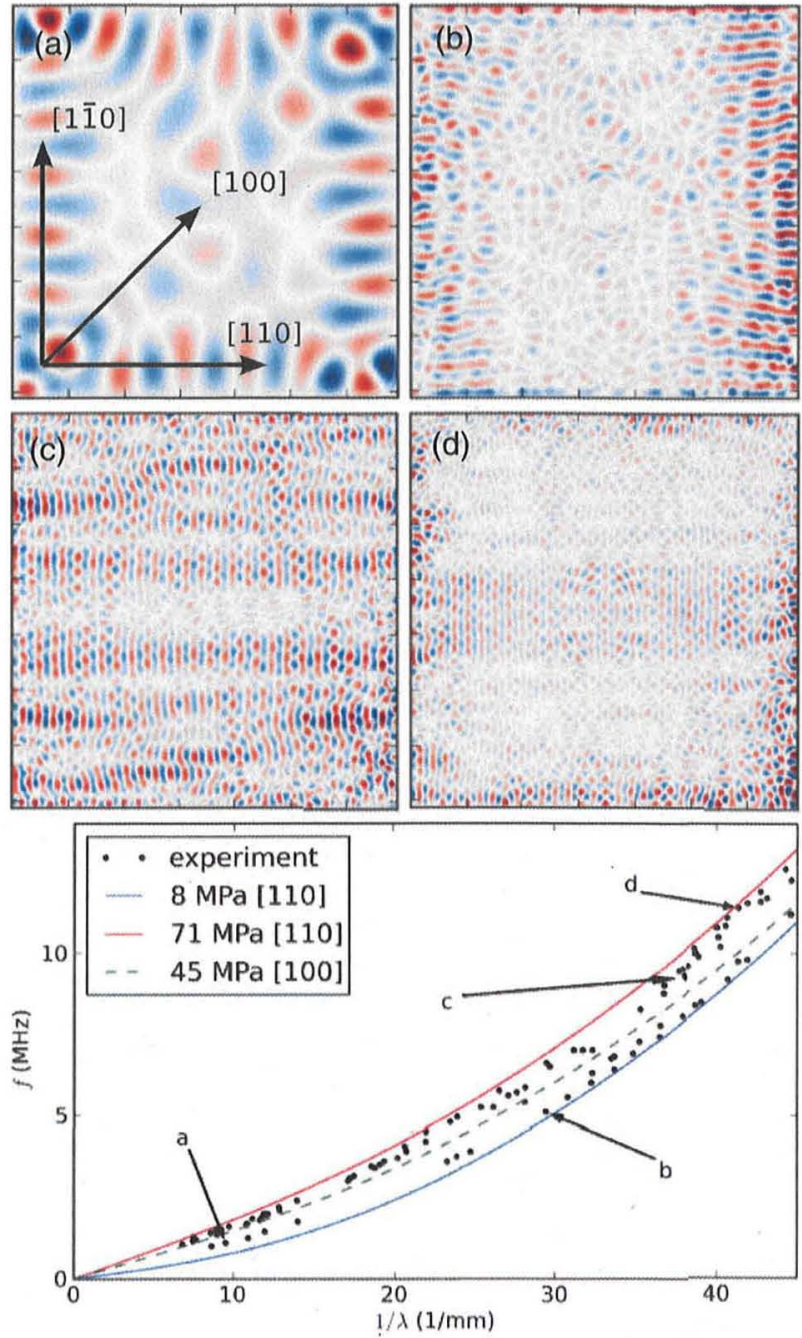

FIG. 2. (Color) (a)-(d) Examples of measured eigenmodes (only the real part is shown) of a membrane with size $714 \mu \mathrm{m} \times$ $691 \mu \mathrm{m} \times 340 \mathrm{~nm}$. The applied pressure difference is $50 \mathrm{mbar}$ and the excitation frequency is varied from 1 to $2 \mathrm{MHz}$. Red and blue denote opposite sign of the phase with respect to the excitation. The darkness of the color is proportional to the deflection amplitude. Lower frequency modes (a) are not localized, higher modes (b)-(d) are usually located along specific paths through the membrane. For comparison with the dispersion relation we distinguish paths along the edge (b), through the center, perpendicular to the edge (d) and completely random patterns (c). (e) Dispersion relation. The black dots are experimental results from the $\vec{k}$-space fit method, and the colored lines are calculated using Eq. (2). The colors correspond to selected paths of wave propagation with varying membrane stress (red: high stress, center of the membrane; blue: low stress, edge of the membrane; dashed green: medium stress, diagonal propagation). The apparent scattering of the experimental data is a result of the localization of modes to paths of higher or lower stress.

with $k_{x l}=l \pi / d_{x}$ and $k_{y m}=m \pi / d_{y} . d_{x}$ and $d_{y}$ are the lateral dimensions of the membrane. Cosine terms are absent because of the fixed boundary conditions. Although we are dealing with 


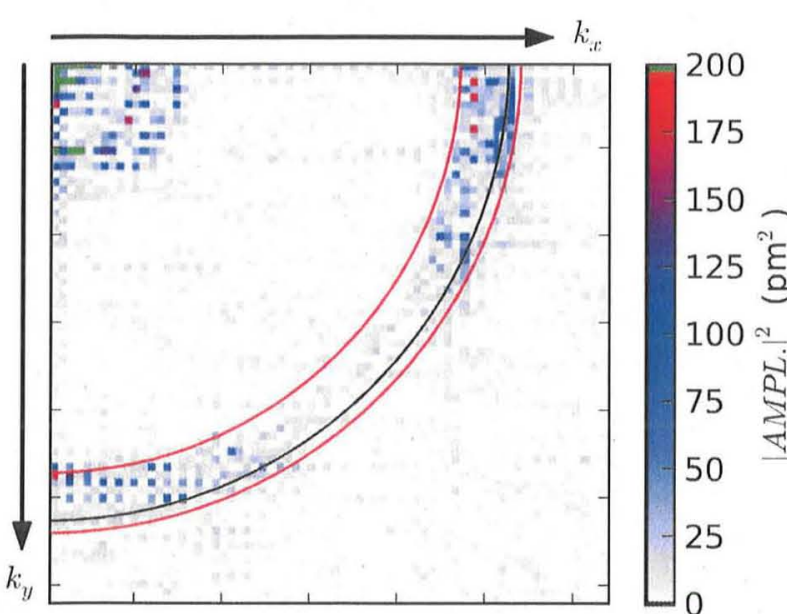

FIG. 3. (Color online) $\vec{k}$-space distribution ( $\left.c_{l m}\right)$ of a mode at 9.8 $\mathrm{MHz}$ (same membrane as in Fig. 2). The color indicates the intensity according to the scale bar at the right side. The solid lines show the fit results for the main contributions [black: peak position; red (gray): half of maximum]. The signal in the upper left corner is an artifact of a lower frequency mode.

standing instead of propagating waves, we can still define a wave vector $\vec{k}=\left(k_{x}, k_{y}\right)$.

Using the $c_{l m}(\omega)$ data, there are two ways to extract a dispersion relation: Either we can find a mean value for $|k|$ for each $\omega$, or we can find the frequency $\omega$, where the component for one specific $\vec{k}$ shows the highest amplitude.

\section{The $\vec{k}$-space fit method}

Since the elastic properties of the (100) plane of silicon are nearly isotropic, the main contributions to the $\vec{k}$-space distribution are found in a circular ring. Fitting a circle to the $c_{l m}(\omega)$ data (black line in Fig. 3), we obtain a data point for an approximately isotropic dispersion relation. Repeating the procedure described above while scanning $\omega_{e x}$, the experimental dispersion relation in Fig. 2(e) is generated. For $\omega_{e x}$ far away from any resonance, the $\vec{k}$-space distribution is dominated by noise. This is most problematic for the first few modes because the spacing between resonance frequencies is high in this regime, limiting this method to frequencies above $\approx 1 \mathrm{MHz}$

\section{E. Maximum-amplitude method}

For the low-frequency part of the dispersion we apply a different method of data analysis. We pick an arbitrary $\vec{k}$ vector and check for which excitation frequency the $\vec{k}$ component of the mode has the highest amplitude (Fig. 4). Repeating this analysis for all the $\vec{k}$ vectors, the experimental data in Fig. 5 is obtained. This method works best for low frequencies $\lesssim 1.5 \mathrm{MHz}$, when the resonance frequencies are well separated, providing a perfect supplement for the $\vec{k}$-space fit method described above. Since the mode for a given frequency is described by a $\vec{k}$-space distribution (Fig. 3 ) rather than a single $\vec{k}$ vector, the $\omega(k)$ data points show apparent scattering as well.

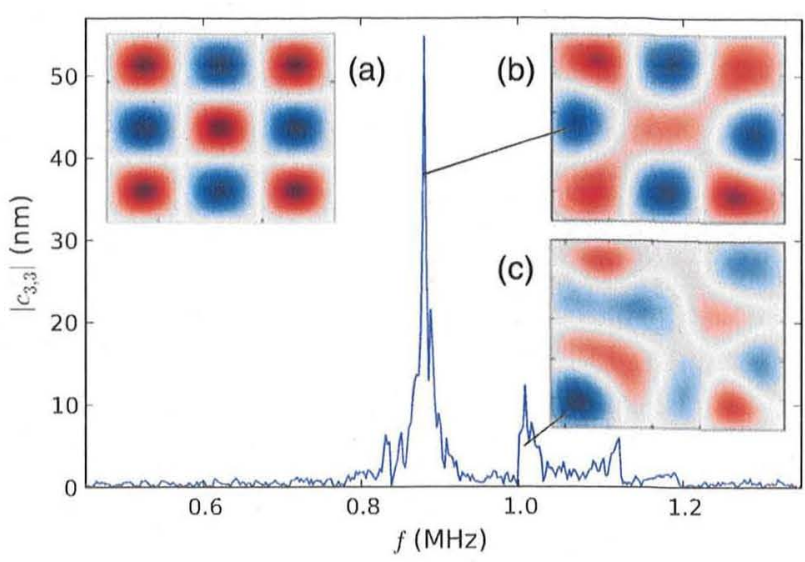

FIG. 4. (Color) The amplitude of the $(l, m)=(3,3)$ component of the measured modes over the excitation frequency $f$. The $(3,3)$ eigenfunction is shown in inset (a). The insets (b) and (c) depict the modes measured at $880 \mathrm{kHz}$ and $1.008 \mathrm{MHz}$ respectively. The mode (b) is a $(3,3)$ eigenmode with only a small $(1,1)$ contribution, resulting in the large peak. (c) is a superposition of higher modes with only a small $(3,3)$ component.

This scattering is part of the nature of the system and should not be mistaken as a measurement error.

\section{F. Discussion of results}

For low frequencies, where wavelengths are comparable to the membrane dimensions, the modes are delocalized

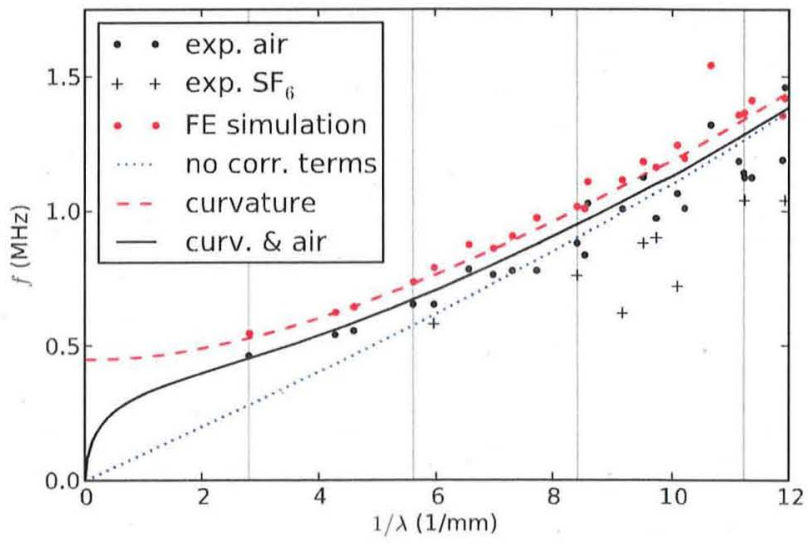

FIG. 5. (Color online) The dispersion relation of membrane bending waves (membrane size $238 \mu \mathrm{m} \times 269 \mu \mathrm{m} \times 337 \mathrm{~nm}$; smaller than the one in Fig. 2). The black dots and pluses are experimental results from the maximum-amplitude method measured in air and $\mathrm{SF}_{6}$ atmospheres respectively; the red (gray) dots are $\mathrm{FE}$ results. The curves show the effect of the correction terms. Unlike in Fig. 2, only curves for diagonal propagation are shown while the curves for maximum and minimum stress are omitted. The gray grid marks the wave numbers of diagonal propagation, where the data points and the shown curves should fit. The dotted blue line depicts the unperturbed result of Eq. (2). The dashed red line takes the effect of static curvature into account using Eq. (3). For the solid black line, the correction factor for air inertia [Eq. (4)] has been applied to the curved membrane dispersion. 
[Fig. 2(a)]. For higher frequencies, the modes are localized along varying paths. Modes along the edge [Fig. 2(b)] tend to have comparatively low frequencies, modes through the center [Fig. 2(d)] have high frequencies, while modes following arbitrary patterns [Fig. 2(c)] show medium frequencies for the same given $|\vec{k}|$. The reason for this is the inhomogeneous stress distribution in the membrane discussed in Sec. III.

\section{G. Finite-elements simulations}

Finite-elements (FE) simulations have been performed using the Comsol Multiphysics software [red (gray) dots in Fig. 5]. The membrane is modeled as a cuboid with four fixed boundaries at the edges and two free boundaries describing the top and the bottom side. A normal force on the bottom side is introduced to describe the pressure difference. In a first step, the pressure-induced static deformation of the membrane is computed. Because of the large static deformations, a nonlinear solver has to be used. In the second step, the equation of motion is linearized around the static solution and the eigenmodes are computed by a linear solver. The mesh consists of approximately 100 points in each lateral direction. In the vertical direction we use four points for the nonlinear and two points for the linear solver. A high number of points in the lateral direction is necessary to get reliable results for small wavelengths, whereas the number of points in the vertical direction has no significant influence on the results.

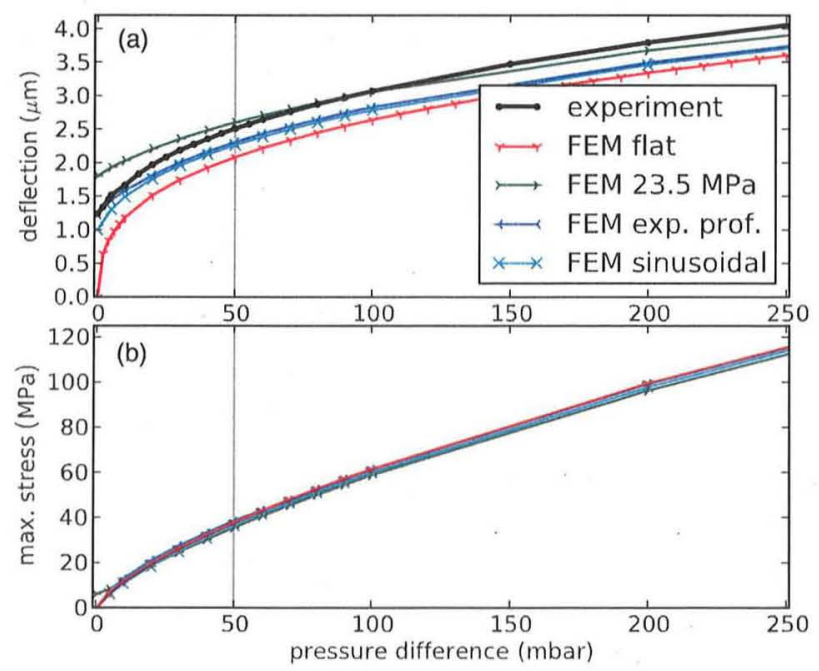

FIG. 6. (Color online) (a) The static deflection of the membrane as a function of the applied pressure difference. Experimental data are shown as well as the results of simulations using different models to take buckling into account. (b) The maximum stress in the membrane as a function of the applied pressure difference. The following FE models have been used: "FEM flat": The membrane is modeled as a cuboid without prestress. Buckling is neglected in this case. "FEM 23.5 MPa": A flat membrane with a compressive prestress of 23.5 $\mathrm{MPa}$, leading to spontaneous buckling compensating the stress. "FEM exp. prof.": The measured profile of the pressure free membrane is used as the equilibrium configuration of the membrane in the simulation. "FEM sinusoidal": A sinusoidal deflection is used as equilibrium configuration of the membrane.
Both the experimental data and the FE simulations show a superlinear dispersion relation starting with a finite frequency for $k \rightarrow 0$. The experimental frequencies are systematically lower as compared to the simulated data. This offset is explained by the inertia of the surrounding media, introducing an additional mass to the membrane not taken into account in the simulations. A detailed discussion of this effect can be found in Sec. IV B of this paper.

To check the validity of the simulations, we compare the simulated static deflection of the membrane as a function of the pressure difference with the experimental data. Using the simplest model possible, a cuboid without prestress, the deflection at 50 mbar from the simulation is $17 \%$ smaller than measured [see Fig. 6(a), lines labeled as "FEM flat" and "experiment"). Regarding the buckling of $1.2 \mu \mathrm{m}$ for zero pressure, this is a reasonably good agreement. We tried several different ways to include buckling in the model, explained in detail in the caption of Fig. 6. All these approaches improve the agreement between simulation and experiment, but none of them results in quantitative agreement for all pressures at once. In Fig. 6(b), the maximum stress is shown for all of the theoretical models investigated. The maximum stress is much less sensitive to buckling than the deflection. The results for different models differ by less than $6 \%$ from the simple flat membrane model. As we will show in the next section, the stress is much more important for the dispersion relation than the deflection, consequently the dispersion relations from our simulations are reliable, although they are calculated neglecting buckling.

\section{ANALYTIC THEORY}

Both prestressed membranes and thin plates have been studied theoretically to great detail in literature. ${ }^{19-21}$ Here the more general case of a prestressed thin plate is discussed.

For prestressed membranes, neglecting bending stiffness, the equation of motion is

$$
h \rho \ddot{z}=F_{z, \text { memb }}=h \sum_{i j} \sigma_{i j} \frac{\partial^{2} z}{\partial x_{i} \partial x_{j}} .
$$

$h$ is the thickness, $z$ the deflection, $F_{z}$ the restoring force density, and $\sigma_{i j}$ the stress tensor of the membrane. The indices $i$ and $j$ cover the lateral directions. For small amplitudes $z$ the stress is constant, given by the static prestress, and therefore the equation of motion is linear. Using the plane-wave ansatz $z \propto$ $e^{i k x-i \omega t}$, the dispersion relation $\omega=\sqrt{\sigma_{x x} \rho} / k$ is obtained. ${ }^{20}$

For thin plates, neglecting both prestress and anisotropy, the restoring force is

$$
F_{z, \text { plate }}=-D \Delta^{2} z
$$

with the bending stiffness $D=E h^{3} / 12\left(1-v^{2}\right)$ using the Young's modulus $E$ and the Poisson ratio v. $\Delta$ denotes the Laplace operator. The dispersion $\omega=\sqrt{D} h \rho / k^{2}$ follows using the same ansatz. ${ }^{20}$ For anisotropic materials, $E$ and $v$ and therefore $D$ depend on the direction of propagation. 
Using the restoring force $F_{z}=F_{z \text {,memb }}+F_{z \text {,plate }}$ we derive the dispersion for a prestressed thin plate:

$$
\omega=\sqrt{\frac{\frac{D}{h} k^{2}+\sigma_{x x}}{\rho}} k .
$$

A more general but less accessible form of the dispersion relation can be found in Ref. 22 .

The only unknown quantity in Eq. (2) is $\sigma_{x x}$, and unfortunately this value is not constant in space. Therefore we use the finite-elements results of $\sigma_{x x}$ to extract the mean values along characteristic paths of wave propagation. These values are indicated in the legend of Fig. 2(e). This way we obtain $\omega(k)$ for waves traveling along the path of maximum stress (perpendicular to the edge through the center), minimum stress (along the edge), and for diagonal propagation. In Fig. 2(e) these extremal dispersion relations are shown in red, blue, and green respectively. The validity of the analysis is verified by the spatial distribution of the real-space images shown in Figs. 2(a)-2(d), which are close to these extremal curves. The interval between these curves is an upper boundary for the uncertainty of the wave number due to inhomogeneous stress. In the experimental data it is visible as the range of scattering in the dispersion relation [Figs. 2(e) and 5], as well as the width of the circular ring in the $\vec{k}$-space map (Fig. 3).

The stressed membrane behavior $\omega \propto k$ and the thin-plate bending behavior $\omega \propto k^{2}$ appear in Eq. (2) as limiting cases for $\operatorname{high}\left(\lambda \gg \lambda_{0}\right)$ and for low $\left(\lambda \ll \lambda_{0}\right)$ wavelengths, respectively. Except for a factor close to 1 , the wavelength $\lambda_{0}$ of the crossover of both regimes is found by equalizing the addends under the square root in Eq. (2):

$$
\lambda_{0}=\sqrt{\frac{E}{\sigma_{x x}}} h .
$$

\section{CORRECTION TERMS}

For small wavelengths (high $k$ ), the analytical expression (2) provides a good description for both the experimental and the FE results (see Fig. 2). For large wavelengths, there is no satisfactory agreement. For very small $k$ in Fig. 5, both the $\mathrm{FE}$ as well as the measured frequencies seem to have a finite limit, while Eq. (2) predicts $\omega(k=0)=0$. This effect will be explained below, taking a static curvature of the membrane into account. The FE method predicts systematically higher frequencies than observed experimentally. This is caused by the inertia of the surrounding gas.

\section{A. Membrane curvature}

As a result of the applied pressure difference, the membranes show a finite curvature. The force needed to bend a curved shell is larger than the force to bend a flat plate, leading to an increase in frequency. The reason for this is the coupling between bending waves and the in-plane waves (longitudinal and shear). For thin shells with small curvature and without prestress, this has been studied theoretically in Refs. 23 and 24. In analogy to the procedure above, we add the stressed-membrane force term to the equation of motion in Ref. 24 and derive the dispersion relation

$$
\omega^{2}=\frac{D}{h \rho} k^{4}+\frac{\sigma_{x x}}{\rho} k^{2}+\omega_{R}^{2}
$$

with

$$
\omega_{R}^{2}=\frac{E}{\rho}\left(\frac{n_{x}^{2}}{R_{y}}+\frac{n_{y}^{2}}{R_{x}}\right)^{2} .
$$

$\vec{n}=\vec{k} / k$ is the normalized vector in propagation direction, and $R_{x}$ and $R_{y}$ are the radii of curvature in $x$ and $y$ directions. Note that this expression is only valid for small curvatures $\left(k \gg 1 / R_{i}\right)$. For frequencies much larger than $\omega_{R}$, the first two terms in Eq. (3) dominate, meaning that membrane curvature is only important for low-frequency modes.

The dispersion relation from Eq. (3) is depicted in Fig. 5 (dashed red line) and shows very good agreement with the FE data. The experimental data is systematically lower in frequency than both Eq. (3) and the FE results. The reason for this is that both of them describe the membrane in vacuum, ${ }^{25}$ neglecting the inertia of the surrounding atmosphere, as we will discuss in the next section.

\section{B. Surrounding fluid}

We estimate the inertia of the air using the simple assumption that the thickness $T=C \lambda$ of the air film in motion is proportional to the wavelength $\lambda$ of the bending wave with a dimensionless constant $C$. The effective density of the membrane is described by $h \rho_{\text {eff }}=h \rho_{\text {memb }}+T \rho_{\text {gas }}$, with the membrane thickness $h$, and the densities of membrane $\rho_{\text {memb }}$ and surrounding gas $\rho_{\text {gas }}$. In the case of different gases on top and bottom side, we assume symmetry in the film thicknesses $h \rho_{\text {eff }}=h \rho_{\text {memb }}+\frac{T}{2} \rho_{\text {gas }, 1}+\frac{T}{2} \rho_{\text {gas }, 2}$. This allows us to treat the two gas system as only one gas with the average density. Using the proportionality $\omega \propto 1 / \sqrt{\rho}$ from Eq. (2) or (3), we conclude

$$
\omega \propto \frac{1}{\sqrt{1+\frac{C \lambda \rho_{\mathrm{gas}}}{h \rho_{\mathrm{memb}}}} .}
$$

Comparing the frequencies $\omega_{1}$ and $\omega_{2}$ of the same mode measured under gas atmospheres with the densities $\rho_{1}$ and $\rho_{2}$ respectively, we isolate $C$ in Eq. (4):

$$
C=\frac{h \rho_{\mathrm{memb}}}{\lambda} \frac{\omega_{2}^{2}-\omega_{1}^{2}}{\rho_{1} \omega_{1}^{2}-\rho_{2} \omega_{2}^{2}} .
$$

Resonance frequencies measured with one side in $\mathrm{SF}_{6}$ and one side in air atmosphere are shown in Fig. 5. The parameter $C$ is calculated for each of these modes using $\rho_{1}=\rho_{\text {Air }}$ and $\rho_{2}=\frac{1}{2}\left(\rho_{\text {Air }}+\rho_{\mathrm{SF}_{6}}\right)$. The mean is $\langle C\rangle=0.66$ with a standard deviation of 0.19 . Using this parameter, we can use the correction factor from Eq. (4) to account for air inertia in the dispersion from Eq. (3). The resulting black line in Fig. 5 is in excellent agreement with the experimental data. For very small wave vectors, corresponding to wavelengths much larger than the size of our membranes, the fluid correction Eq. (4) and therefore the frequency approaches zero. In this regime our approach is no longer valid because the motion is dominated 
by the fluid instead of the membrane, comparable to the motion of a flag in the wind.

\section{CONCLUSION AND SUMMARY}

We have studied the vibrational modes of 340-nm-thick silicon membranes using optical profilometry, FE simulations, and an analytic model. Starting from experimental data in the real space and time domains, we calculate the dispersion relation. The possibility of observing the phenomenon both in real space and in $\vec{k}$ space at the same time, allows us to obtain a very detailed understanding of the system. The physics of the system is governed by two regimes: For low frequencies the nature of the system corresponds to that of a drum head, and for high frequencies thin-plate bending forces dominate the vibrational behavior. We derived correction terms to the analytical description to account for the effects of finite curvature of the membrane and for the inertia of the fluid surrounding the membrane. Including these terms, we obtain excellent agreement between our analytical theory, the FE simulations, as well as the experimental results. Our findings pave the way for tailoring this kind of nanoscale membrane to the requirements of applications relying on particular properties of the vibrational excitations.

\section{ACKNOWLEDGMENTS}

This work was funded by the DFG through SFB767 and the Excellence Initiative. We thank E. Barretto, A. Bruchhausen, T. Dekorsy, A. Erbe, P. Leiderer, P. Nielaba, and the members of the Nanomechanics Discussion Group of SFB767 for valuable discussions. Technical and experimental contributions of S. Diesch, A. Fischer and, M. Schmotz are gratefully acknowledged. *reimar.waitz@uni-konstanz.de

${ }^{\dagger}$ Robert Bosch GmBH, D-70839 Gerlingen-Schillerhöhe, Germany.

${ }^{1}$ G. Sberveglieri, W. Hellmich, and G. Müller, Microsyst. Technol. 3, 183 (1997).

${ }^{2}$ D. Ciarlo, Biomed. Microdevices 4, 63 (2002).

${ }^{3}$ T. Morkved, W. Lopes, J. Hahm, S. Sibener, and H. Jaeger, Polymer 39, 3871 (1998).

${ }^{4}$ T. Krauss and R. De la Rue, Prog. Quantum Electron. 23, 51 (1999).

${ }^{5}$ S. Tomljenovic-Hanic, A. D. Greentree, C. M. de Sterke, and S. Prawer, Opt. Express 17, 6465 (2009).

${ }^{6}$ R. Waitz, O. Schecker, and E. Scheer, Rev. Sci. Instrum. 79, 093901 (2008).

${ }^{7}$ M. Schmotz, P. Bookjans, E. Scheer, and P. Leiderer, Rev. Sci. Instrum. 81, 114903 (2010)

${ }^{8}$ J. Park, H. Qin, M. Scalf, R. T. Hilger, M. S. Westphall, L. M. Smith, and R. H. Blick, Nano Lett. 11, 3681 (2011).

${ }^{9}$ C. C. Striemer, T. R. Gaborski, J. L. McGrath, and P. M. Fauchet, Nature (London) 445, 749 (2007).

${ }^{10} \mathrm{~J}$. D. Thompson, B. M. Zwickl, A. M. Jayich, F. Marquardt, S. M. Girvin, and J. G. E. Harris, Nature (London) 452, 900 (2008).

${ }^{11}$ D. J. Wilson, C. A. Regal, S. B. Papp, and H. J. Kimble, Phys. Rev. Lett. 103, 207204 (2009).

${ }^{12}$ F. Hudert, A. Bruchhausen, D. Issenmann, O. Schecker, R. Waitz, A. Erbe, E. Scheer, T. Dekorsy, A. Mlayah, and J.-R. Huntzinger, Phys. Rev. B 79, 201307 (2009).

${ }^{13}$ C. M. Torres, A. Zwick, F. Poinsotte, J. Groenen, M. Prunnila, J. Ahopelto, A. Mlayah, and V. Paillard, Phys. Status Solidi C 1, 2609 (2004)

${ }^{14}$ J. C. Meyer, A. K. Geim, M. I. Katsnelson, K. S. Novoselov, T. J. Booth, and S. Roth, Nature (London) 446, 60 (2007).
${ }^{15}$ S. Petitgrand, R. Yahiaoui, K. Danaie, A. Bosseboeuf, and J. P. Gilles, Opt. Laser Eng. 36, 77 (2001).

${ }^{16} \mathrm{~J}$. Butschke, A. Ehrmann, E. Haugeneder, M. Irmscher, R. Kaesmaier, K. Kragler, F. Letzkus, H. Loeschner, J. Mathuni, I. W. Rangelow, C. Reuter, F. Shi, and R. Springer, Proc. SPIE 3665, 20 (1999).

${ }^{17}$ V. Ziebart, O. Paul, and H. Baltes, J. Microelectromech. Syst, 8, 423 (1999).

${ }^{18}$ P. Murray and G. F. Carey, J. Appl. Phys. 65, 3667 (1989).

${ }^{19}$ A. Cleland, Foundations of Nanomechanics: From Solid-State Theory to Device Applications (Springer Verlag, Berlin, 2003).

${ }^{20}$ L. D. Landau and E. M. Lifshitz, Lehrbuch der Theoretischen Physik, Band VII Elastizitätstheorie (Akademie Verlag, Berlin, 1991).

${ }^{21} \mathrm{~K}$. Graff, Wave Motion in Elastic Solids (Dover, Mineola, NY, 1975).

${ }^{22}$ E. Nolde, L. Prikazchikova, and G. Rogerson, J. Elast. 75, 1 (2004).

${ }^{23}$ A. D. Pierce, J. Vib. Acoust. 115, 384 (1993).

${ }^{24}$ A. N. Norris and D. A. Rebinsky, J. Vib. Acoust. 116, 457 (1994).

${ }^{25}$ For a more direct test of the mechanics of the pure membrane, experiments in vacuum would obviously be very helpful. Unfortunately this is not easy to do with the setup presented here. The interference objectives used for optical profilometry are usually calculated for use without a cover glass. Simply inserting a vacuum window into the optical path destroys coherence, so a custom-made objective with a built-in correction for the window is necessary. Additionally, for measurements without tensile prestress an alternative stress control mechanism is needed in vacuum, since a pressure difference is no longer available. 